\title{
Exactly solvable cellular automaton traffic jam model
}

\author{
Michael J. Kearney* \\ School of Electronics and Physical Sciences, University of Surrey, Guildford, Surrey GU2 7XH, United Kingdom
}

(Received 25 August 2006; published 26 December 2006; publisher error corrected 5 January 2007)

\begin{abstract}
A detailed study is undertaken of the $v_{\max }=1$ limit of the cellular automaton traffic model proposed by Nagel and Paczuski [Phys. Rev. E 51, 2909 (1995)]. The model allows one to analyze the behavior of a traffic jam initiated in an otherwise freely flowing stream of traffic. By mapping onto a discrete-time queueing system, itself related to various problems encountered in lattice combinatorics, exact results are presented in relation to the jam lifetime, the maximum jam length, and the jam mass (the space-time cluster size or integrated vehicle waiting time), both in terms of the critical and the off-critical behavior. This sets existing scaling results in their natural context and also provides several other interesting results in addition.
\end{abstract}

DOI: 10.1103/PhysRevE.74.061115

PACS number(s): 02.50.-r, 05.40.-a, 45.70.Vn

\section{INTRODUCTION}

Traffic flow may be viewed as an example of a driven many particle system [1,2]. Models based on probabilistic cellular automata have proven especially useful in the study of such systems and, in the context of traffic flow, have helped our understanding of complex phenomena such as jamming [3-8]. Much of this work has relied extensively on simulations and scaling arguments. By contrast, the number of exact analytic results known is comparatively small, although there have been some notable successes, e.g., $[4,6,8]$. Exact results are always welcome, even for simple models, since they provide a benchmark against which one can compare the behavior of more complicated models.

In this paper we revisit the $v_{\max }=1$ limit of a traffic model proposed by Nagel and Paczuski [5], itself a variant of the widely studied model proposed by Nagel and Schreckenberg [3]. The objective is to quantify in detail the statistics of a single jam initiated by the random perturbation of an otherwise freely flowing system. Although much is known already about this model through the use of semiquantitative random-walk arguments [5,9], a complete analysis seems to be lacking. The present paper seeks to address this, providing several interesting results in the process.

An important feature of the model is that it exhibits critical behavior. Thus when the inflow to a given jam derives from the outflow of another jam, and both inflow and outflow are identical in a statistical sense, the jam lifetime distribution $P(T>t)$ has a power-law tail. For the $v_{\max }=1$ limit studied below the jams remain compact and do not branch, whereupon the evolving jam length may be viewed as a random walk whose first-passage time defines the jam lifetime. Since $P(T>t) \sim t^{-1 / 2}$ for an unbiased random walk, this establishes that the jam lifetime probability $P(T=t) \sim t^{-3 / 2}$ in the critical state $[5,9]$. At the qualitative level one may extend this line of reasoning to consider other variables associated with the jam. Thus since the typical excursion of an unbiased random walk at time $t$ scales as $t^{1 / 2}$, the maximum length of the jam during its lifetime should scale as $L \sim T^{1 / 2}$. This suggests that $P(L>l) \sim l^{-1}$ or $P(L=l) \sim l^{-2}$ in

*Electronic address: m.j.kearney@surrey.ac.uk the critical state. Further, the jam mass, which is the integrated vehicle waiting time of all the vehicles involved in the jam during its lifetime, should scale as $M \sim \int_{0}^{T} t^{1 / 2} d t \sim T^{3 / 2}$. This implies that $P(M>m) \sim m^{-1 / 3}$ or $P(M=m) \sim m^{-4 / 3}$ in the critical state. Such arguments are compelling and ultimately correct, but they are not entirely satisfactory. They are also difficult to generalize to the off-critical case, when the jam is initiated in a traffic flow whose average vehicle density is above or below the critical value (see below for details). This corresponds to the jam length executing a biased rather than an unbiased random walk.

In what follows, exact results are obtained in relation to the jam lifetime, the maximum jam length and the jam mass, in both the critical and the off-critical cases. This is achieved by mapping the model onto a discrete-time queueing system which is amenable to analysis using generating function techniques [10]. One benefit is that existing scaling results may now be seen in their full and natural context. Among the results presented, the off-critical behavior of the jam mass is shown to have a particularly nontrivial asymptotic form. This suggests that further studies of this quantity would be welcome for more complicated traffic models.

The paper is organized as follows. In Sec. II, the model is defined. In Sec. III, the jam lifetime probability is derived, followed by the maximum jam length probability. The nearcritical behavior of the expected maximum jam length is also identified. A two-parameter probability generating function is then introduced, which satisfies a given nonlinear functional equation, from which the asymptotic behavior of the jam mass probability can be ascertained. Finally, the results are discussed in Sec. IV.

\section{MODEL}

The model is simple to define [5]. Vehicles occupy sites on an infinite one-dimensional lattice. Each vehicle has a specified velocity $v$, which is either 0 or 1 , and vehicles move from left to right. At the start of a given time step the velocity of each vehicle is updated, in parallel, according to the following rules: (i) If $v=1$ and the adjacent site to the right is empty, the velocity stays at $v=1$. (ii) If the adjacent site to the right is occupied, then the velocity is set to $v=0$. (iii) If $v=0$ and the adjacent site to the right is empty, then 


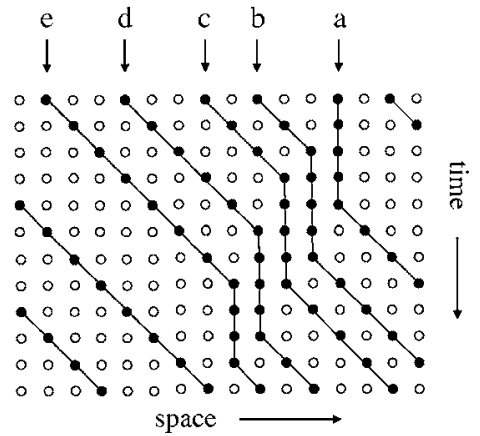

FIG. 1. The evolution of a traffic jam caused by stopping vehicle "a" at time $t=0$. Vehicles are shown as solid circles. In this example all vehicles are freely moving again after ten time steps, thus the jam lifetime $T=10$. The number of vertical "bonds" in the space-time cluster denoting stationary vehicles defines the jam mass, $M=19$.

the velocity increases to $v=1$ with probability $p$, otherwise it stays at $v=0$. During the remainder of the time step the position of each vehicle is then updated, in parallel, according to (iv) The vehicle moves forward $v$ steps. There are equivalent ways of representing the above rules which are more compact, but not necessarily more clear. To set the model into context, rule (i) is the so-called cruise control limit of the Nagel-Schreckenberg model with $v_{\max }=1[3,5]$. This rule distinguishes the model from the asymmetric exclusion process (ASEP) with fully parallel dynamics, for which exact results are known for both periodic [6] and open $[8,11]$ boundary conditions. Rule (ii) prevents vehicles from crashing, a generic feature of models with exclusion dynamics or hard-particle interactions. Rule (iii) implies that vehicles are slow to start, a feature which also appears in different guises in other models $[7,12,13]$. In the context of actual traffic flow, all these behavioral patterns are considered to be realistic $[1,2]$.

A jam consists of a queue of motionless vehicles occupying adjacent sites, and we consider that a jam has resolved itself the instant all vehicles have been updated to $v=1$. To study the behavior of a single jam we choose an initial, freeflowing configuration of vehicles as follows. Moving along the lattice from, say, left to right, sites are occupied with probability $p^{\prime}$ if the preceding site is empty, else the site is left empty, so that no two vehicles occupy adjacent sites. The average density of vehicles is thus $p^{\prime} /\left(1+p^{\prime}\right)$. Each vehicle is then assigned the value $v=1$. Such a configuration will continue to flow freely in the absence of external perturbations, so to initiate a traffic jam a vehicle is chosen at random and its velocity is set to zero. The lifetime of the resulting jam is the time it takes until all the cars are flowing freely again. An example is shown in Fig. 1.

There are two sources of randomness in the model. First, the vehicle at the head of the jam accelerates away with probability $p$, as specified by rule (iii). Second, the choice of initial configuration means that vehicles join the back of the jam with probability $p^{\prime}$. One can equally well consider these vehicles to have originated from a large jam far to the left, where vehicles accelerate away with probability $p^{\prime}$ rather than $p$. By such means one can study the off-critical behav-

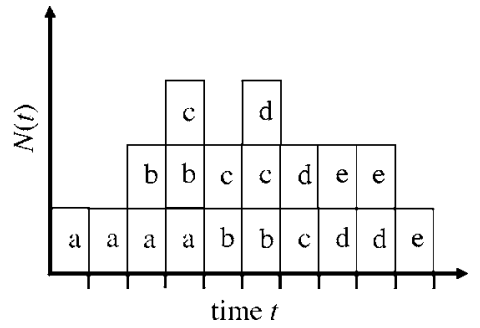

FIG. 2. A queueing representation of the jam shown in Fig. 1, showing the "place in line" of each of the five vehicles involved in the jam throughout its lifetime. The maximum jam length $L=3$, and the integrated vehicle waiting time $M=19$.

ior, $p^{\prime} \neq p$, as well as the critical behavior, $p^{\prime}=p$. Studies of the expected jam lifetime in a similar model were presented in [13]. It should be noted that a slightly different interpretation of when a jam has resolved itself was used in [13], although this has no bearing on the large scale asymptotic behavior deduced in either case.

\section{ANALYSIS}

\section{A. Jam lifetime}

An alternative representation of the jam in Fig. 1 is shown in Fig. 2, wherein the queueing nature of the behavior is made clear. The queue transition probabilities are given by $P_{+}=p^{\prime} q, P_{-}=p q^{\prime}$, and $P_{0}=p p^{\prime}+q q^{\prime}$, where $q \equiv 1-p$ and $q^{\prime} \equiv 1-p^{\prime}$. Since the initial vehicle is stopped at time $t=0$ with probability 1 , the probability of a lifetime- 1 jam is given by $P(T=1)=P_{-}$, while the probability of a lifetime- 2 jam is given by $P(T=2)=P_{0} P_{-}$. For $T \geqslant 3$ we have

$$
P(T=t)=P_{0} P(T=t-1)+P_{+} \sum_{k=1}^{t-2} P(T=t-1-k) P(T=k),
$$

where the last term captures the fact that a length- 2 jam must first become a length-1 jam before it finally resolves itself. Similar ideas were presented in [14] based on the observation that the evolving jam length traces a Motzkin path. From Eq. (1), it is easy to show that the generating function $G(x) \equiv \Sigma_{t} P(T=t) x^{t}$ obeys

$$
G(x)=P_{-} x+P_{0} x G(x)+P_{+} x G(x)^{2}
$$

so that

$$
G(x)=\frac{1-P_{0} x-\sqrt{\left(1-P_{0} x\right)^{2}-4 P_{+} P_{-} x^{2}}}{2 P_{+} x} .
$$

The model admits, for certain parameter choices, the possibility of an infinite jam. This needs to be handled with care. The probability of an infinite jam occurring is $P(T=\infty) \equiv 1-\lim _{x \rightarrow 1} G(x)$. From Eq. (3) one finds that $P(T=\infty)=0$ when $p^{\prime} \leqslant p$; however, $P(T=\infty)=1-P_{-} / P_{+}>0$ when $p^{\prime}>p$. Thus the expected jam lifetime for $p^{\prime}<p$ is given by $\langle T\rangle \equiv d G(x) /\left.d x\right|_{x=1}=1 /\left(p-p^{\prime}\right)$, while $\langle T\rangle=\infty$ when $p^{\prime}>p$. On the other hand, the expected jam lifetime when 
$p^{\prime}>p$ conditional on the jam being finite is given by $\langle T\rangle_{\mathrm{c}} \equiv\left(P_{+} / P_{-}\right) d G(x) /\left.d x\right|_{x=1}=1 /\left(p^{\prime}-p\right)$. Quite generally, the duality transform $p^{\prime} \leftrightarrow p$ maps results for $p^{\prime}<p$ into results for $p^{\prime}>p$ given that the jam is finite $[10,15]$, so hereafter we only consider moments for $p^{\prime}<p$. The expected jam lifetime diverges when $p^{\prime}=p$ and evidently the line $p^{\prime}=p$ is critical.

For $x<x_{c} \equiv\left(P_{0}+2 \sqrt{P_{+} P_{-}}\right)^{-1}$ the right hand side of Eq. (3) may be expanded as a convergent power series in $x$ whose coefficients determine the lifetime probability $P(T=t)$ for $t<\infty$. A useful result in this regard is to note the defining equation

$$
\frac{1}{\sqrt{1-2 z x+x^{2}}} \equiv \sum_{n=0}^{\infty} L_{n}(z) x^{n}
$$

where $L_{n}(z)$ is the $n$th Legendre polynomial [16]. Simple manipulations of Eq. (3) then yield

$$
P(T=t)=\frac{P_{0}^{t+1}}{2(t+1) P_{+} \xi^{t+1}}\left[\xi L_{t}(\xi)-L_{t-1}(\xi)\right]
$$

where $\quad \xi=\left(1-4 P_{+} P_{-} / P_{0}^{2}\right)^{-1 / 2} \geqslant 1$. As $L_{t}(\xi)$ $=\left(2^{t} t !\right)^{-1} d^{t} / d \xi^{t}\left[\left(\xi^{2}-1\right)^{t}\right]$ there is also a slightly more compact expression,

$$
P(T=t)=\frac{2 P_{-} P_{0}^{t-1}}{t(t+1) \xi^{t-1}} \frac{d L_{t}(\xi)}{d \xi} .
$$

These results give the jam lifetime probability exactly for any finite value of $t$ and for all values of $p, p^{\prime}$. The special cases where $p, p^{\prime}=0,1$, when $\xi=1$, may be handled by noting that $d L_{t}(\xi) /\left.d \xi\right|_{\xi=1}=t(t+1) / 2$. For example, as $p^{\prime} \rightarrow 0$ one finds that $P(T=t) \rightarrow p q^{t-1}$. This, as expected, is the probability that the initially stopped vehicle will start moving again in exactly $t$ steps, thus ending the jam. What is of particular interest, however, is the asymptotic behavior as $t \rightarrow \infty$ in the neighborhood of the critical line, such that $\left|p-p^{\prime}\right|<\varepsilon$ where $\varepsilon$ is suitably small. For $t$ integer and large one has from the theory of Legendre functions

$$
L_{t}(z)=\frac{1}{\sqrt{\pi}} \frac{\Gamma(t+1 / 2)}{\Gamma(t+1)} \frac{e^{(t+1) \alpha(z)}}{\left(e^{2 \alpha(z)}-1\right)^{1 / 2}}\left[1+O\left(\frac{1}{t}\right)\right],
$$

where $\alpha(z)=\cosh ^{-1} z>0$ [16]. It then follows from Eq. (5) that in the neighborhood of the critical line as $t \rightarrow \infty$

$$
P(T=t) \sim \frac{1}{\sqrt{4 \pi p q}} t^{-3 / 2} \exp \left(-\frac{\left|p-p^{\prime}\right|^{2}}{4 p q} t\right) .
$$

Thus one observes the expected power law scaling $P(T=t)$ $\sim t^{-3 / 2}$ on the critical line itself [5]. Slightly off the critical line this is augmented by a simple exponential decay controlled by a cutoff time $t_{\mathrm{co}} \approx 4 p q /\left|p-p^{\prime}\right|^{2}$, a scaling which was also identified in [5]. The present analysis, however, goes further in that Eq. (5) is universally valid. It is worth stating that well away from the critical line $t_{\text {co }}$ will be small, whereupon large finite jams are very rare. Of course, when $p^{\prime}>p$ there is a nonzero probability of finding a jam whose lifetime is infinite.

\section{B. Maximum jam length}

The maximum length attained by the jam during its lifetime is given by the random variable $L \equiv \max \{N(t): t$ $=0,1, \ldots, T\}$, where $N(t)$ is the number of vehicles in the jam at time $t$. Thus $L$ is the extremum of a set of strongly correlated random variables, a subject of interest in many other contexts also; see, e.g., [17]. It is actually quite straightforward to derive the maximum jam length probability $P(L=l)$. Let $\hat{P}(l \mid n)$ be the probability that a jam, whose initial length $n>0$, never grows to be larger than some fixed value $l$, i.e., $\hat{P}(l \mid n) \equiv P(L \leqslant l \mid n)$. The strategy is to solve for general $n$ and then set $n=1$ since the jam starts with a single vehicle stopping. The Markov nature of the process implies a simple backward recursion for $\hat{P}(l \mid n)$;

$$
\hat{P}(l \mid n)=P_{-} \hat{P}(l \mid n-1)+P_{0} \hat{P}(l \mid n)+P_{+} \hat{P}(l \mid n+1)
$$

with boundary conditions $\hat{P}(l \mid 0)=1$ and $\hat{P}(l \mid l+1)=0$. The method of solution is standard and one finds that

$$
\begin{gathered}
\hat{P}(l \mid n)=\frac{1-\alpha^{l+1-n}}{1-\alpha^{l+1}} ; \quad \alpha \neq 1, \\
\hat{P}(l \mid n)=1-\frac{n}{l+1} ; \quad \alpha=1
\end{gathered}
$$

for $n \leqslant l$ and $\alpha=P_{+} / P_{-}$. Noting that $P(L=l) \equiv \hat{P}(l \mid n=1)$ $-\hat{P}(l-1 \mid n=1)$ gives

$$
\begin{gathered}
P(L=l)=\frac{\alpha^{l-1}(1-\alpha)^{2}}{\left(1-\alpha^{l}\right)\left(1-\alpha^{l+1}\right)} ; \quad \alpha \neq 1, \\
P(L=l)=\frac{1}{l(l+1)} ; \quad \alpha=1 .
\end{gathered}
$$

These results hold for finite values of $l$. The probability of an infinite jam is given by $P(L=\infty) \equiv 1-\lim _{l \rightarrow \infty} \hat{P}(l \mid n=1)$. From Eq. (10) this is zero for $\alpha<1$ and $1-1 / \alpha$ for $\alpha>1$, i.e., zero for $p^{\prime}<p$ and $1-P_{-} / P_{+}$for $p^{\prime}>p$, as was found previously by considering the jam lifetime.

It follows that on the critical line, where $\alpha=1, P(L=l)$ $\sim l^{-2}$ as $l \rightarrow \infty$. As discussed in the Introduction this may be inferred from the fact that $P(T=t) \sim t^{-3 / 2}$. Interestingly the critical behavior is completely independent of the value of $p$. Near but not on the critical line such that $0<\left|p-p^{\prime}\right|<\varepsilon$ one has for $l \rightarrow \infty$

$$
P(L=l) \sim\left(\frac{\left|p-p^{\prime}\right|}{p q}\right)^{2} \exp \left(-\frac{\left|p-p^{\prime}\right|}{p q} l\right) .
$$

It is important to emphasize that one cannot set $p^{\prime}=p$ in Eq. (12) to recover the critical scaling behavior for $P(L=l)$, as one could in Eq. (8) for $P(T=t)$. In other words, the order in which the limits $p^{\prime} \rightarrow p$ and $l \rightarrow \infty$ are taken is now significant. As a corollary, one cannot "derive" Eq. (12) starting from Eq. (8) and assuming the "plausible" scaling $L \propto\left|p-p^{\prime}\right| T$. An interesting supplementary question concerns the nature of the divergence of the expected maximum jam 
length $\langle L\rangle$ near the critical line. After various elementary manipulations one may show that for $p^{\prime}<p$

$$
\langle L\rangle \equiv \sum_{l=1}^{\infty} l P(L=l)=1+(1-\alpha) \sum_{l=1}^{\infty} \frac{\alpha^{l}}{1-\alpha^{l+1}} .
$$

There is no simple way to carry out the summation explicitly; however, rigorous bounding arguments based on replacing the summation by an integral show that

$$
\langle L\rangle=\frac{(1-\alpha)}{\alpha \ln \alpha} \ln (1-\alpha)+C_{\alpha}
$$

for all $0<\alpha<1$ with $0<C_{\alpha}<1$. This is sufficient to establish that near the critical line, where $\alpha \approx 1$, the divergence of $\langle L\rangle$ is logarithmic

$$
\langle L\rangle=\ln \left(\frac{p q}{\left|p-p^{\prime}\right|}\right)\left[1+O\left(\left|p-p^{\prime}\right|\right)\right] .
$$

The slow divergence supports the idea that most jams never grow to be particularly long (excluding, of course, the infinite jams which occur when $p^{\prime}>p$ ). If $N_{\mathrm{v}}$ denotes the total number of vehicles that participate in the jam throughout its lifetime, it is relatively easy to show that $\left\langle N_{\mathrm{v}}\right\rangle=p /\left(p-p^{\prime}\right)$ for $p^{\prime}<p$. The stronger divergence of $\left\langle N_{\mathrm{v}}\right\rangle$ as $p^{\prime} \rightarrow p$ as compared to $\langle L\rangle$ emphasizes the fact that, typically, $N_{\mathrm{v}} \gg L$ for large jams. In other words, many more vehicles will typically pass through a large jam during its lifetime than will be found in the jam at any one time.

\section{Jam mass}

The jam mass is a considerably more difficult quantity to evaluate than either the jam lifetime or the maximum jam length. The jam mass is defined to be the size of the spacetime cluster associated with the stationary vehicles, as shown in Fig. 1. It is equivalent to the integrated waiting time of all the vehicles involved in the jam as shown in Fig. 2, which establishes its relevance. To make progress, consider the probability $P(t, m)$ that a given jam has lifetime $T=t$ and mass $M=m$. Related to $P(t, m)$ we have a two-parameter generating function $G(x, y)$ such that

$$
G(x, y) \equiv \sum_{t, m} P(t, m) x^{t} y^{m} .
$$

One can show, based on the analysis of the discrete-time queueing system presented in [10], that $G(x, y)$ obeys a nonlinear functional equation,

$$
G(x, y)=P_{-} x y+P_{0} x y G(x, y)+P_{+} x y G(x, y) G(x y, y) .
$$

The derivation of Eq. (17) draws inspiration from the study of a number of problems in lattice combinatorics; see, e.g., $[18,19]$, and in particular is intimately related to the enumeration of staircase polygons [20-23]. It should be noted that the jam lifetime probability, $P(T=t) \equiv \Sigma_{m} P(t, m)$, has the generating function $G(x, 1) \equiv G(x)$, upon which Eq. (17) reduces to Eq. (2) in a satisfying way.
The jam mass probability, $P(M=m) \equiv \Sigma_{t} P(t, m)$, on the other hand, has the generating function $G(1, y)$, which is much harder to determine. Indeed, the formal solution of Eq. (17) for $G(1, y)$, expressible in terms of $q$ series [21], is hopelessly complicated as regards trying to determine $P(M=m)$ exactly. This is despite the fact that one can easily derive the expected jam mass $\langle M\rangle$ exactly from Eq. (17), in conjunction with Eq. (3), namely $\langle M\rangle \equiv \partial G(1, y) /\left.\partial y\right|_{y=1}$ $=p q^{\prime} /\left(p-p^{\prime}\right)^{2}$ for $p^{\prime}<p$.

It is possible, however, to identify the asymptotic form of $G(1, y)$ as $y \rightarrow 1^{-}$, from which the asymptotic form of $P(M=m)$ as $m \rightarrow \infty$ may be determined. By adapting the analysis given in [21] one can derive a contour integral representation of the generating function from which one can eventually show as $y \rightarrow 1^{-}$that

$$
\begin{aligned}
G(1, y)= & \frac{1-P_{0}}{2 P_{+}}+\frac{\left|p-p^{\prime}\right|}{2 P_{+} \lambda^{1 / 3}}(1-y)^{1 / 3} \frac{\mathrm{Ai}^{\prime}\left[\lambda^{2 / 3}(1-y)^{-2 / 3}\right]}{\operatorname{Ai}\left[\lambda^{2 / 3}(1-y)^{-2 / 3}\right]} \\
& {[1+O(1-y)], }
\end{aligned}
$$

where $\operatorname{Ai}(z)$ is the Airy function, $\operatorname{Ai}^{\prime}(z)$ is its derivative, and

$$
\lambda=\frac{3}{4}\left|\ln \left(q q^{\prime}\right) \ln \left(\frac{p^{\prime}}{p}\right)+2 \mathrm{Li}_{2}\left(p^{\prime}\right)-2 \mathrm{Li}_{2}(p)\right|,
$$

where $\operatorname{Li}_{2}(z)$ is the dilogarithm function. These results are uniformly valid over the whole $p, p^{\prime}$ domain, and they are clearly nontrivial in terms of their structure. It is therefore useful to provide a simple, heuristic derivation of Eq. (18) which is valid near the critical line where $\lambda \approx\left|p-p^{\prime}\right|^{3} / 8(p q)^{2}$. Following the arguments presented in [20-23], by inspecting the solution for $G(x, 1)$, given by Eq. (3), one anticipates that $G(x, y)$ will exhibit a tricritical scaling in the neighborhood of the point $\left(x=x_{c}, y=1\right)$ governed by a crossover scaling function $F(t)$;

$$
G(x, y) \sim \frac{1-P_{0} x}{2 P_{+} x}+\frac{(1-y)^{\theta}}{2 P_{+} x} F\left(\frac{x_{c}-x}{(1-y)^{\varphi}}\right),
$$

where it is understood that $x \approx x_{c}$ and $y \approx 1$ and $\theta, \varphi$ are scaling exponents to be determined. As a reminder, the square-root singularity in $G(x, 1)$ occurs when $x=x_{c}$, which defines $x_{c} \equiv\left(P_{0}+2 \sqrt{P_{+} P_{-}}\right)^{-1}$. Writing $x=x_{c}-t(1-y)^{\varphi}$, we now consider the simultaneous limits $y \rightarrow 1^{-}$and $x \rightarrow x_{c}^{-}$such that $t$ remains fixed. Inserting Eq. (20) into Eq. (17), expanding to leading order, and equating powers of $(1-y)$, one finds a nontrivial solution for $F(t)$ if and only if $\theta=1 / 3$ and $\varphi=2 / 3$, whereupon

$$
F(t)^{2}+x_{c}\left(1-P_{0} x_{c}\right) \frac{d F(t)}{d t}-\frac{2\left(1-P_{0} x_{c}\right)}{x_{c}} t=0 .
$$

This first-order, nonlinear Riccati equation has the solution

$$
F(t)=x_{c}\left(1-P_{0} x_{c}\right) \frac{d}{d t} \ln \operatorname{Ai}\left(\frac{2^{1 / 3}}{x_{c}\left(1-P_{0} x_{c}\right)^{1 / 3}} t\right) .
$$

Near the critical line one has $x_{c} \approx 1+\left|p-p^{\prime}\right|^{2} / 4 p q+\ldots$; in other words, $x_{c} \approx 1$. It is then permissible to set $x=1$ in Eq. (20) so that near the critical line for $y \approx 1$ one recovers Eq. (18) with $\lambda \approx\left|p-p^{\prime}\right|^{3} / 8(p q)^{2}$. 
One can quickly establish from Eq. (18) the asymptotic behavior of the jam mass probability on the critical line as $m \rightarrow \infty$. This is determined by the prefactor $(1-y)^{1 / 3}$. Taking the limit $\lambda \rightarrow 0$ and noting that $\mathrm{Ai}^{\prime}(0) / \mathrm{Ai}(0)$ $=-3^{1 / 3} \Gamma\left(\frac{2}{3}\right) / \Gamma\left(\frac{1}{3}\right)$;

$$
\begin{aligned}
P(M=m) & \sim \frac{3^{-2 / 3}}{\Gamma(1 / 3)(p q)^{1 / 3}}\left(\frac{\Gamma(m-1 / 3)}{m !}\right) \\
& \sim \frac{3^{-2 / 3}}{\Gamma(1 / 3)(p q)^{1 / 3}} m^{-4 / 3} .
\end{aligned}
$$

The critical behavior $P(M=m) \sim m^{-4 / 3}$ was given in the Introduction on the basis of a simple scaling argument, and in Eq. (23) the prefactor is identified. To establish the offcritical behavior in such a direct manner is more difficult. However, one can expand Eq. (18) near the critical line $p^{\prime} \approx p$ as $y \rightarrow 1^{-}$in powers of $(1-y)$;

$$
G(1, y) \sim 1+\sum_{n=1}^{\infty}(-1)^{n} 2^{3 n} K_{n} \frac{(p q)^{2 n-1}}{\left|p-p^{\prime}\right|^{3 n-1}}(1-y)^{n},
$$

where the coefficients $K_{n}$ obey a well-known quadratic recursion relation [19]

$$
K_{n}=\left(\frac{3 n-4}{4}\right) K_{n-1}+\sum_{j=1}^{n-1} K_{j} K_{n-j}
$$

with $K_{1}=1 / 8$. It was proved in [19] that $\lim _{n \rightarrow \infty}\left(\frac{4}{3}\right)^{n} K_{n} /(n$ $-1) !=\frac{1}{2 \pi}$, so it follows from Eq. (24) that the moments of the jam mass satisfy

$$
\left.\lim _{\substack{p^{\prime} \rightarrow p \\ k \rightarrow \infty}}\left\langle M^{k}\right\rangle \sim \frac{\partial^{k} G(1, y)}{\partial y^{k}}\right|_{y=1} \sim \frac{1}{2 \pi} 6^{k} k !(k-1) ! \frac{(p q)^{2 k-1}}{\left|p-p^{\prime}\right|^{3 k-1}} .
$$

We know from earlier that $\langle M\rangle \approx p q /\left|p-p^{\prime}\right|^{2}$ when $p^{\prime} \approx p$, so Eq. (26) is quite accurate even for $k=1$. From Eq. (26) one can deduce the asymptotic behavior of the jam mass probability near the critical line as $m \rightarrow \infty$, which is given by [10]

$$
\begin{aligned}
P(M=m) & \sim \frac{1}{2 \sqrt{\pi}}\left(\frac{1}{6}\right)^{1 / 4} \frac{\left|p-p^{\prime}\right|^{7 / 4}}{(p q)^{3 / 2}} m^{-3 / 4} \\
& \exp \left[-\left(\frac{2}{3}\right)^{1 / 2} \frac{\left|p-p^{\prime}\right|^{3 / 2}}{p q} m^{1 / 2}\right] .
\end{aligned}
$$

As was found before when discussing the maximum jam length one cannot set $p^{\prime}=p$ in Eq. (27) to recover the critical behavior given by Eq. (23). Nor can one "derive" the scaling behavior of Eq. (27) starting from Eq. (8), although assuming the "plausible" scaling $M \propto L T \propto\left|p-p^{\prime}\right| T^{2}$ does at least hint at the origin of the stretched-exponential tail seen in Eq. (27). The long-tailed nature of Eq. (27) has implications for the likelihood of occurrence of jams which have a large mass or a large integrated vehicle waiting time. Thus even in this, the simplest of models, the off-critical behavior for this quantity is highly nontrivial.

\section{DISCUSSION}

The model studied in this paper is simplistic, and probably too simplistic to be truly representative of real traffic scenarios. For example, one may legitimately argue that one loses something essential by having a model in which the jams are forced to remain compact. The results should, however, hint at what one might expect to find in more realistic situations. For the jam lifetime probability and the maximum jam length probability it has been possible to find exact results valid over the whole $p, p^{\prime}$ domain, given by Eqs. (5) and (11) respectively. One interesting finding is the logarithmic divergence of the expected maximum jam length, Eq. (15).

For the more complicated jam mass probability it has not been possible to find a solution valid over the whole $p, p^{\prime}$ domain. However, it has been possible to determine the exact asymptotic behavior as $m \rightarrow \infty$ for the critical case, Eq. (23), and for the near-critical case, Eq. (27), and in many respects this asymptotic regime is the most important one. The behavior is far from trivial and it would be interesting to see how this manifests itself in more complicated models, accepting that there is no universal definition of jam mass that is applicable to every model. It seems clear, however, that the concept of an integrated vehicle waiting time is a useful one and, based on the findings for this simple model, it should provide additional insights into the behavior of traffic jams beyond that which can be simply deduced from, say, the jam lifetime alone. Certainly from an operational point of view the integrated vehicle waiting time is a useful parameter with which to characterize the associated cost and inconvenience of traffic jams.

As a final remark, the asymptotic scalings given above are broadly insensitive to the discrete nature of the model and their functional form may therefore be studied by considering the first-passage process for a drifted Brownian motion [24]. This does not, however, detract from the merits of having a direct solution of the cellular automaton model, which is the primary motivation for the present work.

\section{ACKNOWLEDGMENTS}

The author would like to thank Satya Majumdar for useful discussions during a stay at the Isaac Newton Institute for Mathematical Sciences, where part of this work was carried out. 
[1] D. Helbing, Rev. Mod. Phys. 73, 1067 (2001).

[2] K. Nagel, Phys. Rev. E 53, 4655 (1996).

[3] K. Nagel and M. Schreckenberg, J. Phys. I 2, 2221 (1992).

[4] A. Schadschneider and M. Schreckenberg, J. Phys. A 26, L679 (1993).

[5] K. Nagel and M. Paczuski, Phys. Rev. E 51, 2909 (1995).

[6] M. Schreckenberg, A. Schadschneider, K. Nagel, and N. Ito, Phys. Rev. E 51, 2939 (1995).

[7] A. Schadschneider and M. Schreckenberg, Ann. Phys. 6, 541 (1997).

[8] M. R. Evans, N. Rajewsky, and E. R. Speer, J. Stat. Phys. 95, 45 (1999).

[9] S. Redner, A Guide to First-Passage Processes (Cambridge University Press, Cambridge, England, 2001).

[10] M. J. Kearney, J. Phys. A 37, 8421 (2004).

[11] J. de Gier and B. Nienhuis, Phys. Rev. E 59, 4899 (1999).

[12] M. Takayasu and H. Takayasu, Fractals 1, 860 (1993).
[13] R. Barlovic, A. Schadschneider, and M. Schreckenberg, Physica A 294, 525 (2001).

[14] R. A. Blythe, W. Janke, D. A. Johnston, and R. Kenna, J. Stat. Mech.: Theory Exp. 2004, P10007.

[15] J. W. Essam, J. Phys. A 22, 4927 (1989).

[16] I. S. Gradshteyn and I. M. Rhyzhik, Tables of Integrals, Series and Products, 5th ed. (Academic, London, 1994).

[17] S. N. Majumdar and A. Comtet, J. Stat. Phys. 119, 777 (2005).

[18] E. Domany and W. Kinzel, Phys. Rev. Lett. 53, 311 (1984).

[19] L. Takacs, Adv. Appl. Probab. 23, 557 (1991).

[20] T. Prellberg and R. Brak, J. Stat. Phys. 78, 701 (1995).

[21] T. Prellberg, J. Phys. A 28, 1289 (1995).

[22] C. Richard, J. Stat. Phys. 108, 459 (2002).

[23] M. J. Kearney, J. Phys. A 35, L731 (2002).

[24] M. J. Kearney and S. N. Majumdar, J. Phys. A 38, 4097 (2005). 